\title{
Fluorescence Micro-tomography of Frozen-hydrated Whole Cells using the Bionanoprobe
}

\author{
S.Chen, ${ }^{1}$ C.Flachenecker, ${ }^{2}$ T.Paunesku, ${ }^{3}$ Y.Yuan, ${ }^{3}$ W.Liu, ${ }^{3}$ J.Deng, ${ }^{4}$ Q.Jin, ${ }^{5}$ R.Wak, ${ }^{5}$ B.Lai, ${ }^{1}$ \\ B.Hornberger, ${ }^{2}$ C.Roehrig, ${ }^{1}$ J.VonOsinski, ${ }^{6}$ M.Bolbat, ${ }^{6}$ J.Maser, ${ }^{1}$ D.Shu, ${ }^{1}$ S.Gleber, ${ }^{1}$ L.Finney, \\ K.Brister, ${ }^{6}$ C.Jacobsen, ${ }^{1,4,5,7}$ G.Woloschak, ${ }^{3}$ S.Vogt ${ }^{1,5}$ \\ ${ }^{1}$ Advanced Photon Source, Argonne National Laboratory, Argonne, IL 60439, USA \\ ${ }^{2}$ Carl Zeiss, Pleasanton, CA 94588, USA \\ ${ }^{3}$ Department of Radiation Oncology, Northwestern University, Chicago, IL 60611, USA \\ ${ }_{5}^{4}$ Applied Physics, Northwestern University, Evanston, IL 60208, USA \\ ${ }^{5}$ Department of Physics and Astronomy, Northwestern University, Evanston, IL 60208, USA \\ ${ }^{6}$ Synchrotron Research Center, Northwestern University, Argonne, IL 60439, USA \\ ${ }^{7}$ Chemistry of Life Processes Institute, Northwestern University, Evanston, IL 60208, USA
}

Hard X-ray fluorescence (XRF) microscopy is one of the most sensitive imaging techniques to study trace elemental distributions in biological specimens, such as tissues and cells. By using the Bionanoprobe (BNP) installed at the Life Sciences Collaboration Access Team (LS-CAT) at the Advanced Photon Source (Fig.1), we are able to study the distributions of elements in frozen-hydrated whole cells with sub-100 nm spatial resolution [1,2]. However, two-dimensional projections of elemental distributions within a sample provide very limited depth information, which often leads to inaccurate interpretation of the sample. This becomes a concern particularly for thick samples with complex three-dimensional structures, such as biological whole cells.

To tackle this issue, we have implemented tomography capabilities at the BNP. Mainly due to the space limitation inside the BNP vacuum chamber, a rotation stage was mounted on top of the stage stack as opposed to being underneath the horizontal $(\mathrm{X}) / \operatorname{vertical}(\mathrm{Y})$ translation stages. As a result, the point of interest (POI) for scanning on the sample usually is not on the stage rotation axis (Fig.2a). Realigning of the POI to the X-ray beam focus is required after each rotation (Fig.2b). To carry out this realignment automatically, a mechanism has been implemented to the operation system. This mechanism calculates the offsets in both the horizontal direction and the direction along the X-ray beam $(Z)$ after each rotation based on two vectors: (1) the vector between the X-ray beam focus and the rotation axis (fixed after initial microscope alignment), and (2) the vector between the POI and the rotation axis (fixed once the sample is mounted). Accordingly, the POI is translated using $\mathrm{X} / \mathrm{Z}$ stages back into the X-ray beam focus. Prior to tomogram reconstruction, projection image alignment, rotation axis correction, and background subtraction are performed, which effectively reduce reconstruction artifacts and improve the tomogram quality.

In addition to the technical details, we will report in this presentation the latest results on the study of three dimensional distributions of nanocomposites within frozen-hydrated whole cells. By examining the samples from a series of time points, we are able to follow the delivery path of the nanocomposites into the cells and determine whether they can target the desired subcellular structure. This study significantly benefits from the use of the tomography technique at the BNP because of its high spatial resolution, high throughput and cryogenic capabilities. The three-dimensional intracellular ultrastructure is well preserved when the cells are maintained in their frozen-hydrated state. The duration of acquiring 
a full tomographic dataset of such a cell is approximately 30 hours, over which no radiation damage is detected.

\section{References}

[1] S. Chen et al., J. of Synchrotron Rad. 21, 66-75 (2014).

[2] Y. Yuan et al., ACS Nano (2013) PubMed PMID: 24219664.

[3] This research was supported by ARRA: NIH/NCRR High End Instrumentation (HEI) grant (1S10RR029272-01). We thank NIH grant 2 R01 for some of the cryogenic sample preparation equipment used. Argonne National Laboratory's work was under US Department of Energy contract DE-AC02-06CH11357.
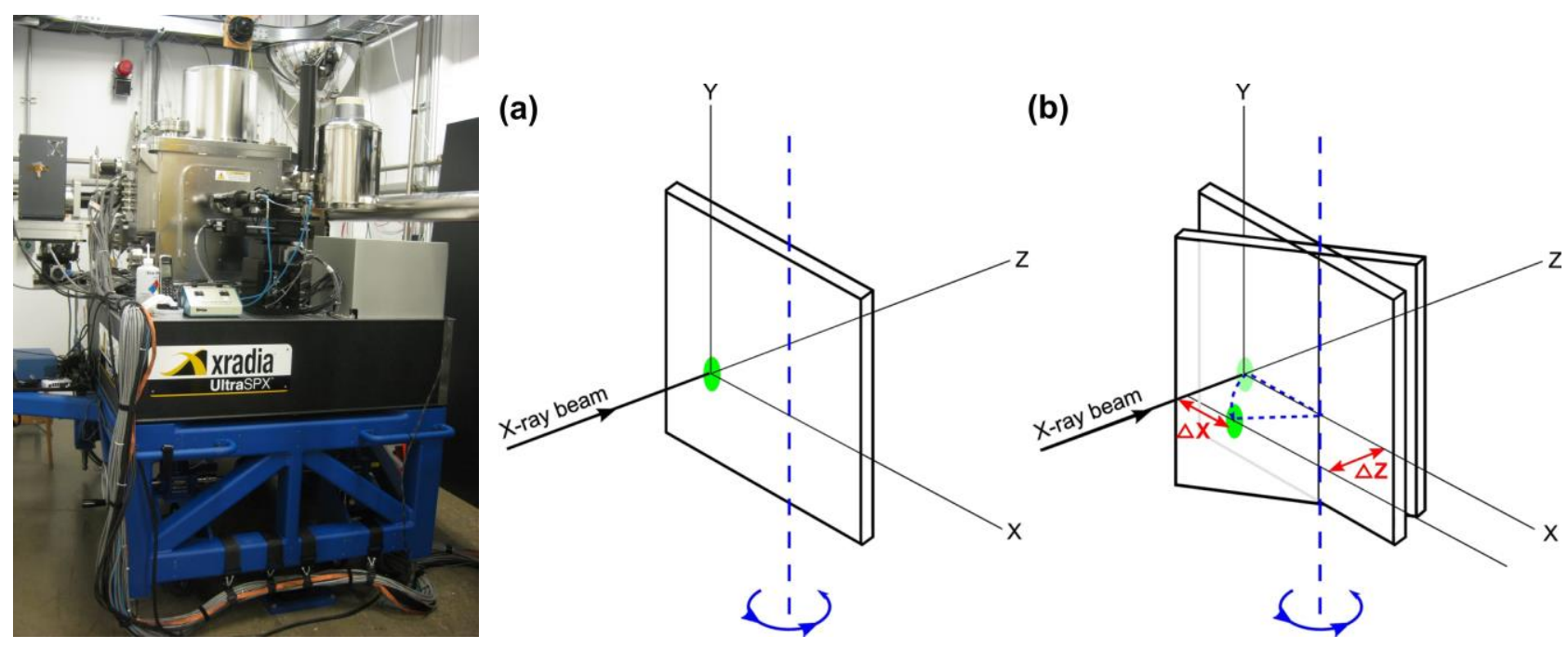

FIG. 1. Picture showing the Bionanoprobe (BNP), which was installed at an undulator beamline at the Life Sciences Collaboration Access Team (LS-CAT) at the Advanced Photon Source. The BNP is a hard $\mathrm{X}$-ray fluorescence nanoprobe dedicated to studying trace elements in frozen biological samples.

FIG. 2. Schematics showing the misalignment of the point of interest (POI, indicated in green) to the X-ray beam focus after a rotation around a vertical axis. (a) The POI is in the X-ray beam focus by initial alignment, which is often not on the rotation axis of the sample stage, indicated by the blue dotted line; and (b) after a rotation, the POI is out of the X-ray beam. The translations are needed in both the horizontal direction by $\Delta \mathrm{x}$ and the direction parallel to the beam by $\Delta \mathrm{z}$ in order to move the POI back into the X-ray beam focus. 